\title{
Screen Printing Reliable Wearable Microstrip Antennas on Rough Textile Substrates
}

\author{
Mahmoud Wagih, Abiodun Komolafe, Russel Torah, Alex S. Weddell, and Steve Beeby \\ School of Electronics and Computer Science, University of Southampton, Southampton, SO17 1BJ, United Kingdom \\ \{mahm1g15; aok1g15; rnt; asw; spb\}@ecs.soton.ac.uk
}

\begin{abstract}
Textile-based antennas are typically realized using conductive fabrics adhered to thick non-woven fabrics such as felt, which are not suited for additive manufacturing due to their roughness. This paper presents screen printing on thin and breathable polyurethane films as a method for realizing etextile antennas without altering the feel of the fabric, demonstrated through a microstrip antenna on standard felt fabric. The fabricated proximity-coupled patch maintains at least 100 $\mathrm{MHz}(4 \%)$ bandwidth from 2.46 to $2.56 \mathrm{GHz}$ regardless of bending and human proximity, $6.6 \mathrm{dBi}$ measured gain, and $69 \%$ radiation efficiency. The robustness of the proposed antenna is demonstrated, for the first time, through the measured radiation properties, showing that the antenna maintains its peak gain and radiation patterns after more than 10,000 bending cycles.
\end{abstract}

\section{INTRODUCTION}

Textile antennas continue to attract significant research interest for wearable communications and RF energy harvesting applications [1], [2]. Microstrip patch antennas are the predominant antenna topology used for communications with an off-body source, owing to their high front-to-back ratio which improves their isolation from the body. Furthermore, several novel textile patch antennas have been proposed for dual-polarization communication, simultaneous information and power transfer [2], and high-isolation [3].

Microstrip antennas need to maintain a thickness of $0.02 \times \lambda$ to achieve a high radiation efficiency. Therefore, patch antennas have typically been demonstrated mostly on non-woven felt fabrics, which exhibit higher thickness and lower $\tan \delta$, making them suitable for RF applications [2], [3], [4], [5]. The aforementioned antennas have been mostly realized using either laser-cut conductive fabrics or conductive threads, both of which require encapsulation using thick polymer (PDMS) to achieve mechanical reliability [6]. In addition, porous nonwoven fabrics are unsuitable for direct screen printing [4], which is widely regarded as one of the most scalable methods for mass producing e-textile systems [7]. Furthermore, while the reliability of textile antennas has been previously investigated based on $S_{11}$ and RFID read-range measurements, the implications of bending and washing on a microstrip antenna's radiation properties remain unclear.

In this paper, a simple fabrication method is presented, enabling additive manufacturing of robust microstrip antennas on textile substrates which are otherwise unsuitable for direct printing. The reliability of the antenna is demonstrated through experimental $S_{11}$ and radiation properties measure-

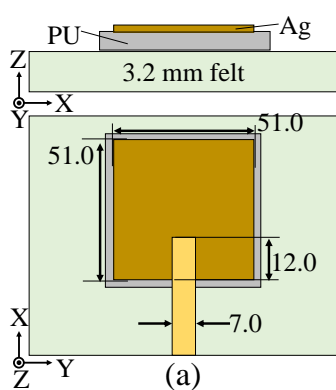

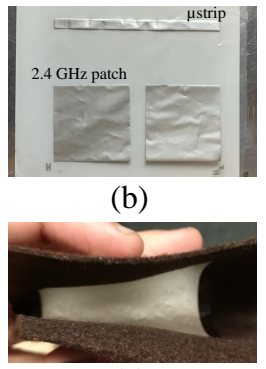

(c)

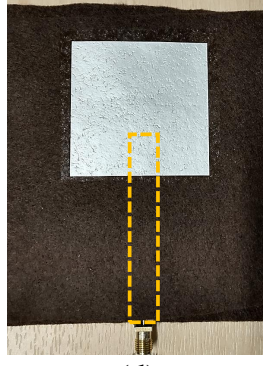

(d)
Fig. 1. Layout and photographs of the screen printed PU textile antenna: (a) dimensions in $\mathrm{mm}$; (b) printed silver microstrip line and patch on PU; (c) demonstration of the patch's flexibility; (d) the connectorized prototype.

ments, showing that the antenna's high radiation efficiency and gain can be maintained after $>10,000$ bending cycles.

\section{Antenna Printing ON PU}

Additively manufactured antennas on textiles have been first proposed using inkjet printing, following the deposition of an interface layer which reduces the fabric's roughness [5]. Nevertheless, this technique was only demonstrated for thin and relatively smooth textiles such as woven polyester/cotton, where a substrate such as felt would not be suitable [4]. The first step in the proposed fabrication method is screen printing the antenna's pattern onto a polyurethane (PU) film, followed by thermal curing at $70^{\circ} \mathrm{C}$ for 8 minutes. The antenna is fabricated using a screen-printable low-temperature Smart Fabric Inks silver ink (TC-C4007). The film in Fig. 11 b), is then heatpressed onto the textile substrate, felt, at $170^{\circ} \mathrm{C}$ for 30 seconds. Unlike [8], where PU was used as an interface prior to printing the antenna, curing the ink twice drastically improves the conductivity, reducing the sheet resistance from 2-5 $\Omega$ /square to $30-50 \mathrm{~m} \Omega / \mathrm{square}$, in line with that of conductive fabrics widely used to realize microstrip antennas [2]. A top PU layer can then be laminated to fully waterproof the antenna.

A proximity-fed microstrip patch antenna for the $2.4 \mathrm{GHz}$ license-free band is fabricated using the proposed technique. The proximity feed is selected as it can improve the bandwidth and matching compared to inset microstrip feeds [2], and enables reliable interconnection without through-textile vias [4]. Fig. 1 shows the layout and photographs of the fabricated microstrip patch antenna. 


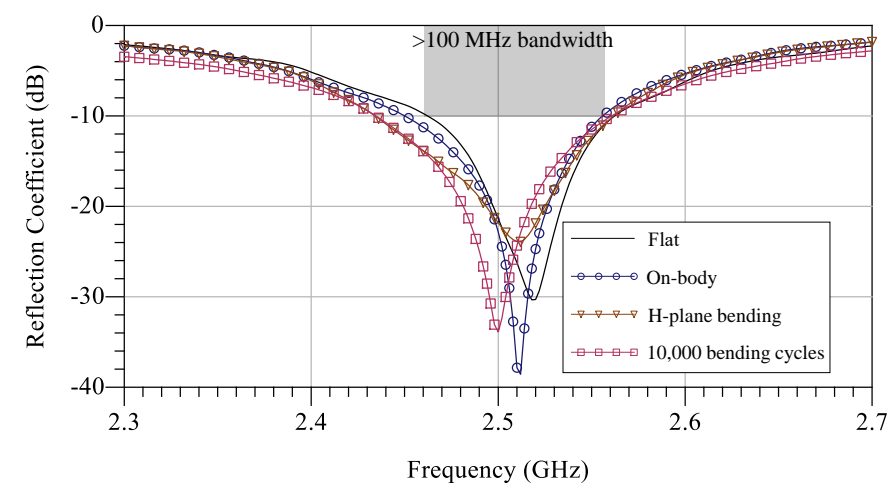

Fig. 2. Measured reflection coefficient of the screen printed textile antenna.

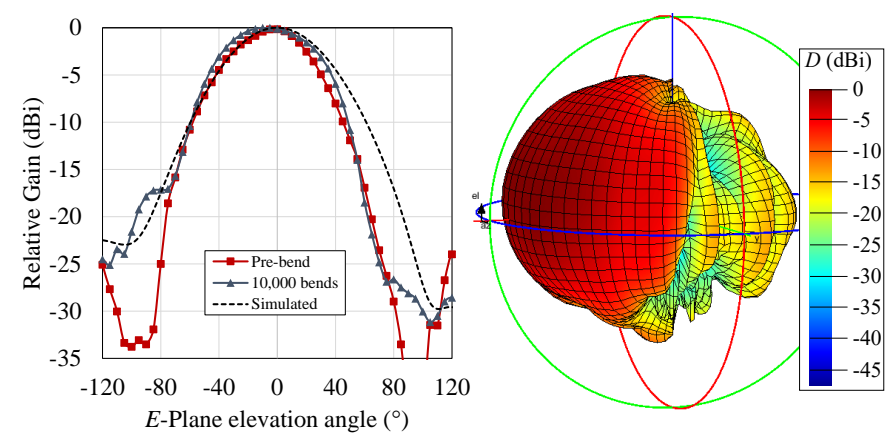

Fig. 3. The simulated and measured $E$-plane patterns of the antenna (left) and the measured $3 \mathrm{D}$ pattern after 10,000 bending cycles (right).

\section{Antenna Simulation And Measurements}

An SMA connector has been soldered onto an adhered copper tape on the feeding microstrip line, to simplify the antenna measurements compared to conductive epoxy. The prototype in Fig. 11.d) was measured using a TOSM-calibrated R\&S ZVB4 VNA. Fig. 2 shows the simulated and measured $S_{11}$, showing over $100 \mathrm{MHz}$ bandwidth from $2.43 \mathrm{GHz}$. Moreover, the antenna's bandwidth is maintained for onbody and while bending. To demonstrate the reliability of the proposed antenna, the antenna was bent repetitively around a $5 \mathrm{~mm}$ radius for 10,000 cycles. As shown in Fig. 2, the $S_{11}$ bandwidth is maintained after the bending test.

The antenna's radiation properties were simulated in CST Microwave Studio based on the measured sheet resistance of the double-cured silver ink of $50 \mathrm{~m} \Omega /$ square, measured using an impedance analyzer. The $3 \mathrm{D}$ radiation patterns were experimentally measured in an indoor echoic environment. Fig. 3 shows the antenna's measured 3D patterns, along with the simulated and measured patterns over the $E$-plane.

Reliable and robust textile antennas have so far only been characterized using their $S_{11}$ response following bending and washing tests [6]. However, the $S_{11}$ alone is not indicative of the antennas' far-field performance, where the radiation properties may have degraded. Following 10,000 bending cycles, the antenna's 3D radiation patterns and gain have been remeasured. As observed on the $E$-plane in Fig. 3 , the antenna maintains its radiation patterns showing its high robustness and suitability for wearable applications.

The gain was measured using the two-antenna method with VNA-based time-domain gating to exclude multi-path effects. The measured gain of the antenna is $6.8 \mathrm{dBi}( \pm 0.5 \mathrm{~dB})$. From the gain to directivity ratio, the antenna maintains a measured $69 \%$ total efficiency at $2.4 \mathrm{GHz}$, in close agreement with the simulated efficiency of $66 \%$. Following the bending tests, the antenna's measured gain is $6.6 \mathrm{dBi}$, showing minimal influence on the antenna's performance. Furthermore, the measured radiation efficiency is in line with that achieved using conductive fabrics on the same substrate [2], [4], demonstrating that printed antennas realized using the proposed method can achieve high efficiency and gain.

\section{Conclusion}

In this paper, a novel fabrication method was proposed for realizing robust printed antennas on textile substrates unsuitable for printing. The antenna's reliability has been investigated through radiation properties and $S_{11}$ measurements, demonstrating a stable gain after more than 10,000 bending cycles. Future work includes washing tests, insertion loss measurements, and investigating different antenna designs.

\section{ACKNOWLEDGEMENT}

This work was supported by the UK EPSRC Grant EP/P010164/1, the H2020-EU.1.4.1.2 grant number: 730957 and H2020-ICT-02-2018 Grant agreement ID: 825339.

\section{REFERENCES}

[1] C.-X. Mao, D. Vital, D. H. Werner, Y. Wu, and S. Bhardwaj, "DualPolarized Embroidered Textile Armband Antenna Array With Omnidirectional Radiation for On-/Off-Body Wearable Applications," IEEE Trans. Antennas Propag., vol. 68 no. 4, pp. 2575 - 2584, 2020.

[2] M. Wagih, G. S. Hilton, A. S. Weddell, and S. Beeby, "Dual-Band DualMode Textile Antenna/Rectenna for Simultaneous Wireless Information and Power Transfer (SWIPT)," IEEE Trans. Antennas Propag., 2021.

[3] C. X. Mao, Y. Zhou, Y. Wu, H. Soewardiman, D. H. Werner, and J. S. Jur, "Low-Profile Strip-Loaded Textile Antenna with Enhanced Bandwidth and Isolation for Full-Duplex Wearable Applications," IEEE Trans. Antennas Propag., vol. 68 no. 9, pp. 6527 - 6537, 2020.

[4] S.-E. Adami, P. Proynov, G. S. Hilton, G. Yang, C. Zhang, D. Zhu, Y. Li, S. P. Beeby, I. J. Craddock, and B. H. Stark, "A Flexible 2.45-GHz Power Harvesting Wristband With Net System Output From $-24.3 \mathrm{dBm}$ of RF Power," IEEE Trans. Microw. Theory Techn., vol. 66 no. 1, pp. 380-395, 2018.

[5] W. G. Whittow, A. Chauraya, J. C. Vardaxoglou, Y. Li, R. Torah, K. Yang, S. Beeby, and J. Tudor, "Inkjet-Printed Microstrip Patch Antennas Realized on Textile for Wearable Applications," IEEE Antennas Wireless Propag. Lett., vol. 13, pp. 71-74, 2014.

[6] R. B. V. B. Simorangkir, Y. Yang, R. M. Hashmi, T. Björninen, K. P. Esselle, and L. Ukkonen, "Polydimethylsiloxane-embedded conductive fabric: Characterization and application for realization of robust passive and active flexible wearable antennas," IEEE Access, vol. 6, pp. 48102 $48112,2018$.

[7] K. Yang, R. Torah, Y. Wei, S. Beeby, and J. Tudor, "Waterproof and durable screen printed silver conductive tracks on textiles," Textile Research Journal, vol. 83, pp. 2023 - 2031, 2013.

[8] I. Martinez, C.-X. Mao, D. Vital, H. Shahariar, D. H. Werner, J. S. Jur, and S. Bhardwaj, "Compact, low-profile and robust textile antennas with improved bandwidth for easy garment integration," IEEE Access, vol. 8, pp. 77 490-77 500, 2020. 